\title{
MEASUREMENT OF PREFERENTIAL FLOW DURING INFILTRATION AND EVAPORATION IN POROUS MEDIA
}

\author{
Papafotiou A. ${ }^{1}$, Schütz C. ${ }^{1}$, Lehmann P. ${ }^{2}$, Vontobel P. ${ }^{3}$, \\ Or D. ${ }^{2}$, and Neuweiler I. ${ }^{1}$ \\ ${ }^{1}$ Leibniz Universität Hannover, Institute of Fluid Mechanics and Environmental Physics in Civil Engineering, \\ 30167 Hannover,Germany,papafotiou@hydromech.uni-hannover.de, schuetz@hydromech.uni-hannover.de, \\ neuweiler@hydromech.uni-hannover.de \\ ${ }^{2}$ Soil and Terrestrial Environmental Physics, ETH Zürich, 8092 Zürich, Switzerland, \\ peter.lehmann@env.ethz.ch,dani.or@env.ethz.ch \\ ${ }^{3}$ Paul Scherrer Institute, 5232 Villigen, Switzerland, peter.vontobel@psi.ch
}

\begin{abstract}
Infiltration and evaporation are governing processes for water exchange between soil and atmosphere. In addition to atmospheric supply or demand, infiltration and evaporation rates are controlled by the material properties of the subsurface and the interplay between capillary, viscous and gravitational forces. This is commonly modeled with semi-empirical approaches using continuum models, such as the Richards equation for unsaturated flow. However, preferential flow phenomena often occur, limiting or even entirely suspending the applicability of continuum-based models. During infiltration, unstable fingers may form in homogeneous or heterogeneous porous media. On the other hand, the evaporation process may be driven by the hydraulic coupling of materials with different hydraulic functions found in heterogeneous systems.

To analyze such preferential flow processes, water distribution was monitored in infiltration and evaporation lab experiments using neutron transmission techniques. Measurements were performed in $2 D$ and $3 D$, using homogeneous and heterogeneous setups. The experimental findings demonstrate the fingering effect in infiltration and how it is influenced by the presence of fine inclusions in coarse background material. During evaporation processes, the hydraulic coupling effect is found to control the evaporation rate, limiting the modeling of water balances between soil and surface based on surface information alone.
\end{abstract}

Key words: preferential, infiltration, evaporation, unsaturated, drying, unstable, fronts, neutron, imaging, radiography, tomography.

\section{Introduction}

A significant part of water exchange between the subsurface and the atmosphere occurs through infiltration and evaporation in the unsaturated zone. Infiltration and evaporation are governed concurrently by relevant flow and transport processes both in the atmosphere and the subsurface, posing thus an inherent complexity when it comes to the description and prediction of such systems. For a given atmospheric supply or demand, infiltration and evaporation processes in the subsurface are controlled by the soil properties and the interplay between capillary, viscous and gravitational forces. 
In order to achieve predictions for such problems, these effects are commonly described by assigning effective medium properties to the soil (ie. permeability), combined with continuum-based models for flow, such as the Richards equation for the unsaturated zone. Nevertheless, continuum-based approaches have certain limitations, as the substitution of real materials with effective media dictates that some processes vanish during the averaging procedure. Such limitations are revealed when preferential flow phenomena arise and need to be accounted for, as a basic feature of such models is the stability of the solution.

A typical example of preferential flow is the formation of unstable wetting fronts during infiltration in initially dry porous media. Instability in wetting fronts is triggered by pore-scale heterogeneity, stemming from different pore sizes and shapes, that determines the local forces and therefore the overall front propagation features in the air-filled pore space. Such effects have therefore been studied on the pore-scale consideration with pore-network models (DiCarlo 2006) and invasion-percolation models (Glass and Yarrington, 1996). On the effective medium scale, preferential flow has been widely investigated with $2 \mathrm{D}$ experiments of infiltration in homogeneous porous media using Hele-Shaw cells (Glass et al., 1989; DiCarlo, 2004). Similar 2D experiments have also been conducted using inclusions of fine material in coarse background, showing that heterogeneity tends to locally eliminate the instability in the inclusions (Hill and Parlange, 1972; Sililo and Tellam, 2000; Rezanezhad et al., 2006). Nevertheless, due to the typically fast propagation of infiltration fronts, monitoring preferential flow in 3D has been limited to delineating unstable wetting fronts and finger diameters (Glass et al., 1990; Tullis and Wright, 2007). Saturation measurements have thus been restricted to $2 \mathrm{D}$ setups that carry a degree of uncertainty related to the influence of the boundaries on the flow process. In the first part of the work presented here we extend saturation measurements to 3D, discussing whether our 2D knowledge on preferential flow is indeed relevant for the 3D space and focussing on the effect of material interfaces. For that purpose, we measure water saturation distribution in a 3D column during unstable wetting for a homogeneous case as well as for a case with fine material inclusions in a coarse background.

Similar limitations of classical continuum models are encountered when preferential flow is induced by evaporation. On the pore scale, evaporation from porous media causes water movement from the larger pores near the drying front to smaller pores near the surface, driven by the capillary pressure difference. This effect, termed as capillary pumping (Yiotis et al., 2001), has extensively been investigated from a pore-scale consideration (Prat, 1993; Prat, 2002; Yiotis et al., 2004). Experimental work is also found on the medium scale, using initially wet or party wet homogeneous porous media subject to evaporation (Shokri et al., 2008). Similarly to the pore-scale pumping effect, the effect of hydraulic coupling has been demonstrated on the medium scale using coupled vertical columns of different materials (Lehmann and Or, 2009). In this case, preferential water flow is induced from the coarse-textured to the fine-textured column through the coupling of the materials, sustaining high evaporation rates through the fine-textured surface for longer periods. These observations indicate that, in order to predict evaporation rates from soils, one needs to account for the subsurface structural features and the emerging preferential flow paths additionally to the classical drying front dynamics considerations. In the second part of this work, we investigate the effect of hydraulic coupling by monitoring water saturation distribution during evaporation from a heterogeneous structure that consists of a tortuous fine-textured inclusion embedded in a coarse background.

\section{Description of experiments}

\subsection{Infiltration experimental setup}

The infiltration experiment was carried out in 3D using an aluminium cylindrical column with 150 
(a)

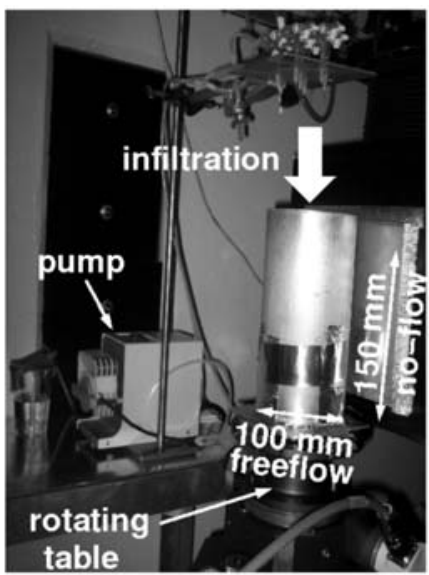

(b)

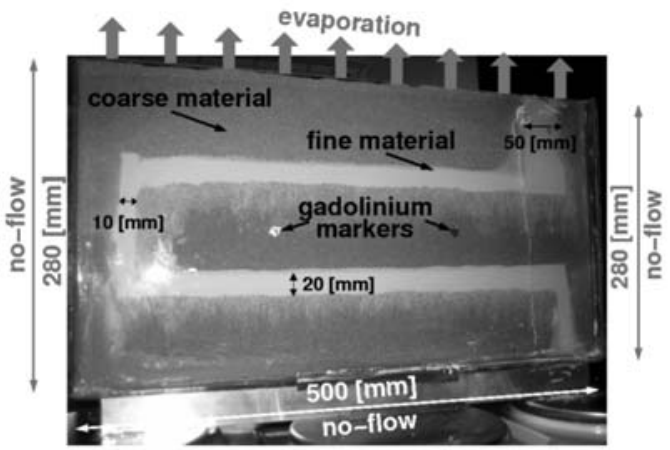

Fig. 1: Experimental setups: infiltration experiments (a), evaporation experiment (b).

mm height and $100 \mathrm{~mm}$ inner diameter (Fig. 1a). The experiment was first performed with a homogeneous packing of a coarse material. Then it was repeated with fine-textured inclusions embedded in the coarse background. The inclusions were lunate-shaped in the horizontal plane and were introduced symmetrically with respect to the centre of the column at $\mathrm{z}=15$ and $30 \mathrm{~mm}$, each having a height of $10 \mathrm{~mm}$. This inclusion configuration left a wide opening of the coarse material towards the bottom. The coarse material chosen was quartz sand with grain sizes ranging from 0.7 to $1.2 \mathrm{~mm}$. Quartz sand with grain sizes from 0.1 to $0.3 \mathrm{~mm}$ was used as a fine material. The sands were packed by simply pouring the particles into the column entirely dry, which also corresponds to the initial condition for the infiltration experiments. The base of the column was perforated to avoid the ponding of water at the bottom and to prevent any air pressure build-up inside the porous medium. A fine metal grid sealed the outlets to keep the sand particles from flowing out with water.

The top of the column was open to the atmosphere and subjected to a constant infiltration rate of 10 $\mathrm{ml} / \mathrm{min}$ (Fig. 1a). The infiltration water mass was delivered equally to the sand surface by distributing the inflow into tubes ending at several injection points. Additionally, the top of the structure was covered with a $20 \mathrm{~mm}$ thick layer of the fine material, to ensure an initial homogeneous spreading of the water mass in this layer through capillarity and prevent the formation of artificial preferential flow paths near the injection points. The bottom boundary was of free-flow type and any water reaching the bottom was collected in an external container positioned under the column.

\subsection{Evaporation experimental setup}

The evaporation experiment was carried out in 2D using a Hele-Shaw cell with $280 \mathrm{~mm}$ height, 500 $\mathrm{mm}$ width and a thickness of $20 \mathrm{~mm}$. The heterogeneous structure consisted of a tortuous fine-textured inclusion connecting bottom and top of the cell in a coarse-textured background (Fig. 1b). As coarse background material, quartz sand with grain sizes ranging from 0.7 to $0.9 \mathrm{~mm}$ was used. The fine material was quartz powder with grain sizes in the range of 0 to $0.06 \mathrm{~mm}$ with a mean value of $0.012 \mathrm{~mm}$ diameter. This resulted in a contrast of the saturated hydraulic conductivity of several orders of magnitude. In order to achieve a fully saturated initial condition, the entire structure was packed under water. The sand was first flushed an immersed in separate glass beakers. Artificial 
variations of porosity during the wet packing were prevented by depositing the wet sand particles from a constant falling distance into the water-filled Hele-Shaw cell and mixing the packed particles each 10-20 mm (Lehmann et al. 2008). Shaping the tortuous inclusion was achieved by placing metal sheets that were slowly shifted after the fine particles had settled. Despite the painstaking procedure, some mixing of the two materials near the boundaries of the inclusion was inevitable due to the large grain size contrast.

The top of the Hele-Shaw cell was open to the atmosphere and acted as the evaporative surface (Fig. 1b). A hair-dryer, blowing with a constant fan speed from a fixed distance towards the evaporative surface, was used to manipulate the evaporation rate. This offers the advantage of a faster evaporation process without having any influence on the water distribution inside the porous medium (Shokri et al., 2008).

\subsection{Measurement techniques}

Water movement inside the porous media was monitored using thermal neutron transmission technology in the NEUTRA station of the Spallation Neutron Source (SINQ) of the Paul Scherrer Institute, Switzerland. The following measurements were performed:

- Infiltration experiments (fast process): 2D width-averaged dynamic water distribution by means of fast radiography in time increments of $4 \mathrm{~s}$ during the front propagation. Additionally, 3D reconstruction of infiltration patterns by means of tomography obtained by rotational scanning in steps of $2^{\circ}$.

- Evaporation experiment (slow process): 2D water distribution by means of slow radiography in time increments of 30 mins for a period of $6.5 \mathrm{~d}$.

Measuring water distribution in porous media with neutron imaging is based on relating water saturation to neutron intensity detected on a scintillator behind the scanned medium. The neutron intensity I passing through quartz sand, water and cell or column walls (ie. glass or aluminium) is

$$
\mathrm{I}=\mathrm{Io} \exp (-\alpha q d q-\alpha \mathrm{wdw}-\alpha c d c)
$$

with the source neutron beam intensity Io, the neutron attenuation coefficients $\alpha \mathrm{q}, \alpha \mathrm{w}, \alpha \mathrm{c}$ and effective thicknesses dq, dw, dc for quartz, water and cell or column wall, respectively. The recorded intensity I was filtered to correct source beam intensity variations and neutron scattering effects. Consequently, the water effective thickness $\mathrm{dw}$ can be deduced by comparing each image (intensity I) to a reference entirely wet or dry image (Iref) that thus has a known water thickness dref equal to dwet or zero.

$$
\mathrm{dw}=\operatorname{dref}-(1 / \alpha \mathrm{w}) \ln (\mathrm{I} / \mathrm{Iref})
$$

In a similar fashion, the coefficient $\alpha$ w can be determined with Eq. (1) using the mean values mwet and mdry of intensities at entirely wet and dry conditions

$$
\alpha \mathrm{w}=-\ln (\text { mwet } / \text { mdry }) / \text { dwet }
$$

Combination of Eqs. (2) and (3) yields the water saturation

$$
\mathrm{Sw}=\mathrm{dw} / \mathrm{dwet}=\mathrm{dref} / \mathrm{dwet}+\ln (\mathrm{I} / \mathrm{Iref}) / \ln (\text { mwet } / \text { mdry })
$$

The term dref/dwet obviously reduces to one or zero, depending on the reference image used.

In the infiltration experiment, the water mass in the system was defined by the water inflow prescribed with the balance. For the evaporation experiment, however, the evaporative mass was, naturally, not 


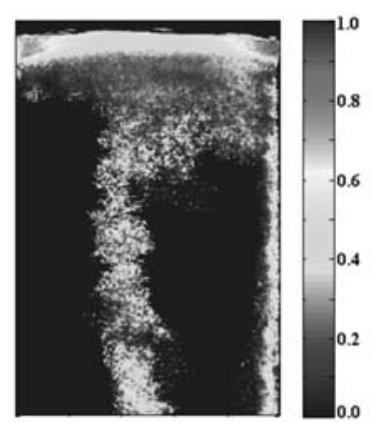

(a)

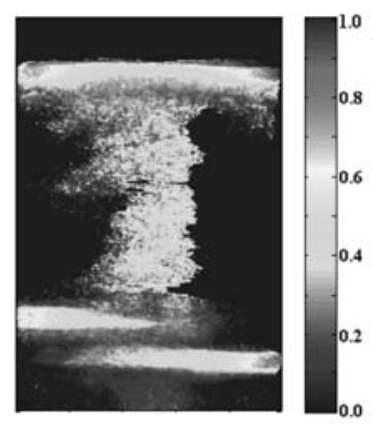

(b)
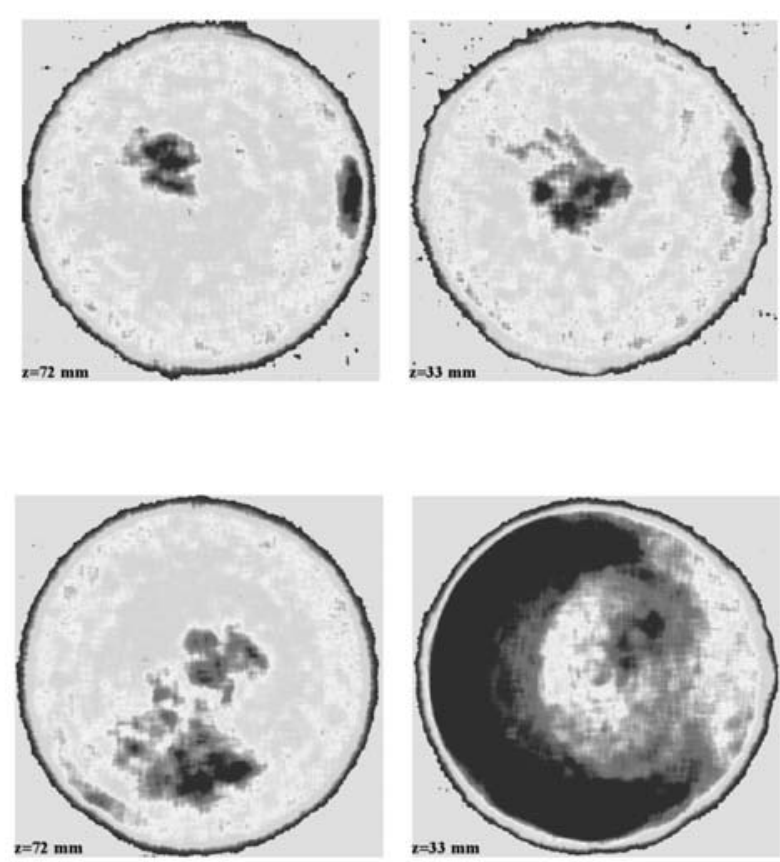

Fig. 2: Finger formation during wetting: (a) in the homogeneously packed coarse material and (b) with lunateshaped inclusions of fine material. The left image illustrates depth-averaged saturation obtained with neutron radiography. Middle and right image qualitatively depict neutron intensities in cross-sections (100 mm diameter) of the column at heights $\mathrm{z}=72$ and $\mathrm{z}=33 \mathrm{~mm}$, respectively.

predefined and therefore the total water mass in the Hele-Shaw cell was recorded continuously with digital balances. This was done for the entire measurement period of $6.5 \mathrm{~d}$ and was also continued after the end of the saturation measurements until $\mathrm{t}=13 \mathrm{~d}$. More details are given in Sect. 3.2.

\section{Results}

\subsection{Infiltration experiments}

With the application of the infiltration rate, water first distributed in the fine material at the top and saturated it. Consequently, preferential flow in the infiltration experiments occured in terms of unstable finger formation in the coarse material (Fig. 2). The fingers formed at the interface between the fine and the coarse material and then vastly propagated through the coarse material towards the bottom of the column, driven by gravity. It must be stated that fingering here was triggered purely by pore-scale effects and was not initiated with any kind of artificial structures or variations in the infiltration rate.

The homogeneous packing (Fig. 2a) resulted in the formation of two fingers. The vertical propagation is illustrated here with a radiography image (left), while cross-sectional neutron intensities delineate the finger area in the column at $\mathrm{z}=72$ and $33 \mathrm{~mm}$ (middle and right image). The first finger came in contact with the wall of the column soon after its formation at the fine-coarse interface and then reached the bottom within $22 \mathrm{~s}$, remaining attached to the wall. Nevertheless, the second fin- 

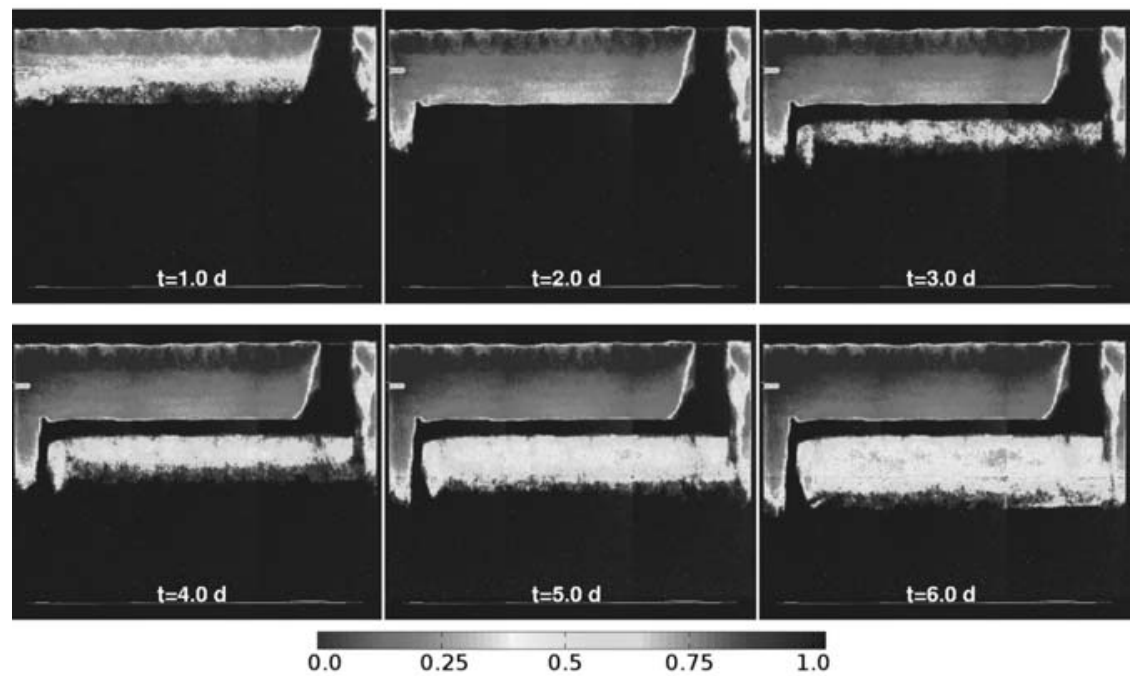

Fig. 3: Water saturation distribution determined with neutron radiography during the evaporation experiment at $\mathrm{t}=1,2,3,4,5$ and 6 days.

ger formed in the middle of the column, reaching the bottom within $36 \mathrm{~s}$. These observations indicate that boundaries can, indeed, introduce preferential pathways for water and possibly create or accelerate preferential flow phenomena at the walls. However, fingering also occurs away from the column walls, demonstrating that unstable wetting is also relevant for 3D applications.

The heterogeneous case (Fig. 2b) demonstrates the formation of a finger and its behaviour in the presence of the two symmetric, lunate-shaped fine material inclusions. Also in this case, the vertical propagation is illustrated with a radiography image (left) while cross-sections are shown at $\mathrm{z}=72$ and $33 \mathrm{~mm}$ (middle and right image). Once more, the finger was formed near the centre of the column (left and middle image), however in this case it was eliminated at the lower region of the column near the fine inclusions (left and right image). This happened despite the fact that the central cross-sectional area was not occupied by fine material due to the symmetric lunate shapes of the inclusions; a configuration that would, in general, allow finger propagation through the centre. However, any hydraulic connection of the finger to the fine material drives water into the inclusion due to the capillary pressure difference, interrupting the finger propagation and forming a smeared-out infiltration front. Through this pronounced pore-scale capillary effect, material interfaces dominate the system and determine the stability of the wetting front. This is visible in the radiography image (left) as well as in cross-sectional image near the inclusions (right). In the cross-section at $\mathrm{z}=33 \mathrm{~mm}$, the finger is still discernible near the centre of the column, as well as its hydraulic connection to the (at this stage wet) upper inclusion. Despite the fact that the infiltration rate from the top was continued, fingering effects underneath the inclusions were not observed even after the inclusions were saturated. However, this presumably also relates to the limited height of the column.

\subsection{Evaporation experiment}

The saturation distribution obtained with neutron radiography during the evaporation experiment is given in Fig. 3 for the times $t=1,2,3,4,5$ and 6 d. The saturation images also allow a straight-forward derivation of the change of total water mass in the porous medium during the evaporation 


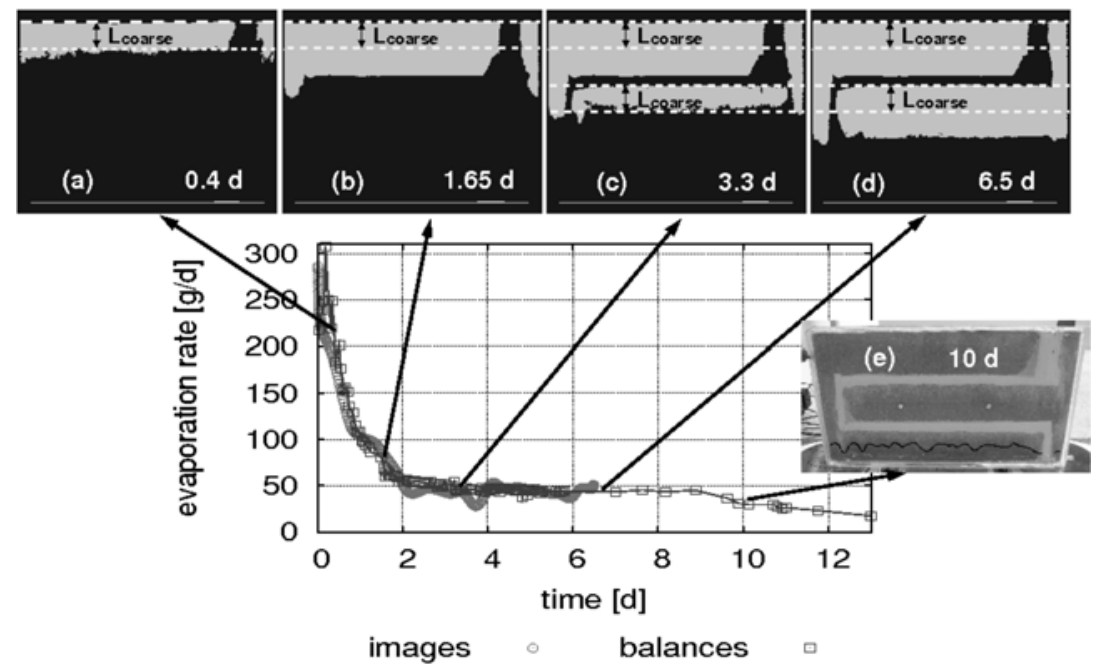

Fig. 4: Evaporation rate determined from the saturation images and the digital balance measurements. Front positions at different phases of the evaporation process $(\mathrm{t}=0.4,1.65,3.3,6.5$ days and qualitatively at $\mathrm{t}=10$ days $)$ are also illustrated.

process. It is thus possible to derive the evaporation rate based on information from the neutron radiography alone. This evaporation rate is compared to the one measured with the digital balances during the experiment in Fig. 4. This procedure is commonly followed as a calibration method for neutron imaging as an additional correction of intensity variation and scattering errors. However, in this case agreement between the radiography-based mass balance and the balance measurements was obtained without any calibration.

The evaporation rate is characterized by three periods: ( 1 ) and initial period from $t=0$ to $t=0.4 \mathrm{~d}$ with rate values fluctuating between 200 and $250 \mathrm{~g} / \mathrm{d}$, (2) a falling rate period from $\mathrm{t}=0.4$ to $\mathrm{t}=1.65 \mathrm{~d}$ with a vast decrease of rate from 250 to $60 \mathrm{~g} / \mathrm{d}$ and (3) a constant rate period from $\mathrm{t}=1.65$ to $\mathrm{t}=6.5$ with slow rate decrease from 60 to $44 \mathrm{~g} / \mathrm{d}$. Additionally, the balance measurements indicate that the rate of $44 \mathrm{~g} / \mathrm{d}$ was maintained until $\mathrm{t}=9 \mathrm{~d}$. Although the evolution of the evaporation rate resembles the classical consideration of a first (high rates) and a second (low rates) stage of evaporation, the origins of this pattern have to be closely examined, accounting for the fact that this rate stems from a coupled system of two materials. The saturation images show that the top of the fine material remained saturated until the end of the experiment, it is thus safe to conclude that the fine material sustained first stage evaporation. This is, however, not the case for the coarse material. An estimation of the drying front depth that initiates the transition from first to second stage evaporation can be given by the characteristic evaporation length Lcoarse of the coarse material (Lehmann et al. 2008). Derivation of the material characteristic lengths and comparison of this information to the deduced drying front images (Fig. 4) reveals that the drying front reaches depth Lcoarse at $t=0.4 \mathrm{~d}$, indicating that this time corresponds to the end of first stage evaporation of the coarse material.

Nevertheless, the structure continued to supply high evaporation rates within the range of 44 to $60 \mathrm{~g} / \mathrm{d}$ until $\mathrm{t}=9 \mathrm{~d}$. This evaporative mass can be attributed to (i) mass that originated from the drying front and reached the surface as vapour diffusion through the coarse material, (ii) first-stage evaporation of the fine material, which, since the fine material remained saturated throughout the entire meas- 
urement, translates to a hydraulic coupling effect of the two materials. The evaporative mass supplied through the vapour diffusion mechanism (i) can be estimated based on Penman's model (Penman 1940), using the medium porosity, the diffusion of vapour in free air, the saturated vapour density at the front, the vapour density at the surface, the water content above the front and the drying front depth. Based on this approach, the maximum diffusive flux (ie. for the front position shown in Fig. $4 \mathrm{~b}$ ) obtained during the second stage evaporation from the coarse material in this setup was found in the order of $0.01 \mathrm{~g} / \mathrm{d}$. This value corresponds to $1 \mathrm{~g} / \mathrm{d} \mathrm{per}^{2}$, a finding that agrees with previous observations on diffusive fluxes during second stage evaporation from porous media (Shokri et al. 2008).

This diffusive flux is negligible compared to the $44 \mathrm{~g} / \mathrm{d}$ evaporation rate measured until $\mathrm{t}=9 \mathrm{~d}$, denoting that the measured rate was mainly sustained through first stage evaporation from the fine material. This strong contrast of contribution of evaporative mass originating on the one hand from the diffusion-driven process and on the other hand from the material coupling, highlights the significance of hydraulic coupling effects during evaporation from heterogeneous porous media. Water is drawn from the coarse material background into the fine material and flows through the tortuous path towards the fine-textured evaporative surface, supplying the evaporative demand. Practically, this effect sustains higher evaporation rates for longer periods than one would predict by neglecting the coupled behaviour of the system. This preferential water flow through the inclusion is driven by the atmospheric demand but is strongly controlled by the contrast of hydraulic properties of the materials as well as the properties of the inclusion geometry, especially with respect to connectivity.

\section{Conclusions}

The first part of the presented work deals with monitoring preferential flow during infiltration in porous media. We present the 3D measurement of water saturation distribution during unstable wetting in initially dry porous media. Two different cases are examined: a homogeneous case using a coarse material and a heterogeneous case with fine material inclusions embedded in the coarse background. The experiments showed that preferential flow phenomena can be significant for 3D problems of infiltration, however, one has to be aware of artificial boundary effects, ie. when investigating such processes in 2D. The existence of fine inclusions eliminated, at least locally, the fingering effect, even though the inclusion configuration used here could, theoretically, allow the finger propagation towards the bottom. This behaviour stems from capillary pressure difference in the materials and reveals that material interfaces play a dominant role in the 3D infiltration process: any hydraulic connection between the finger and the inclusion (even through few pores) can stabilize the wetting front.

The second part presents 2D measurements of water saturation distribution during preferential flow induced by hydraulic coupling of materials during evaporation from heterogeneous porous media. The structure under examination consisted of a tortuous fine-textured inclusion, connecting the bottom to the evaporative surface at the top, embedded in a coarse background. The experiment revealed the significance of the coupling effect: even though the end of first stage evaporation for the background material was reached soon, the structure continued to supply high evaporation rates through the fine-textured surface until the entire background material was practically dried out. The observations made here reveal that evaporation rates can be strongly underestimated when neglecting the coupling behaviour of materials in the subsurface. Therefore, in order to achieve predictive modelling of evaporation from soils, it is necessary to account for subsurface structural features of heterogeneity.

\section{Acknowledgments}

The authors would like to thank the Deutsche Forschungsgemeinschaft (DFG) for supporting this 
work within the project Multi-Scale Interfaces in Unsaturated Soil under the grants Ne 824/6-1 and Ne 824/8-1.

\section{References}

DiCarlo, D.A., 2004. Experimental measurements of saturation overshoot on infiltration. Water Resources Research 40(4), W04215.

DiCarlo, D.A., 2006. Quantitative network model predictions of saturation behind infiltration fronts and comparison with experiments. Water Resources Research 42(7), W07408.

Glass, R.J., Steenhuis, T.S. and Parlange, J.Y., 1989. Mechanism for finger persistence in homogeneous unsaturated porous media: theory and verification. Soil Science 148, pp. 60-70.

Glass, R.J., Cann, S., King, J., Baily, N., Parlange, J.Y. and Steenhuis, T.S., 1990. Wetting front instability in unsaturated porous media: a three-dimensional study in initially dry sand. Transport in Porous Media 5, pp. 247-268.

Glass, R.J. and Yarrington, L., 1996. Simulation of gravity fingering in porous media using a modified invasion-percolation model. Geoderma 70, pp. 231-252.

Hill, D.E. and Parlange, J.Y., 1972. Wetting front instability in layered soils. Soil Sci. Soc. Am. Proc. 36, pp. 697-702.

Lehmann, P., Assouline, S., and Or, D., 2008. Characteristic lengths affecting evaporative drying of porous media. Physical Review E 77(5), 056309.

Lehmann, P. and Or, D., 2009. Evaporation and capillary coupling across vertical textural contrasts in porous media. Physical Review E 80,046318.

Penman, H.L., 1940. Gas and vapor movements in the soil: I. The diffusion of vapors through porous media. Journal of Agricultural Science 30, pp. 437-462.

Prat, M., 1993. Percolation model of drying under isothermal conditions in porous media. International Journal of Multiphase Flow 19(4), pp. 691-704.

Prat, M., 2002. Recent advances in pore-scale models for drying of porous media. Chemical Engineering Journal 86, pp. 153-164.

Rezanezhad, F., Vogel, H.-J., and Roth, K., 2006. Experimental study of fingered flow through initially dry sand. Hydrol. Earth Syst. Sci. Discuss. 3, pp. 2595-2620.

Shokri, N., Lehmann, P., Vontobel, P., and Or, D., 2008. Drying front and water content dynamics during evaporation from sand delineated by neutron radiography. Water Resources Research 44, W06418.

Sililo, O.T.N. and Tellam, J.H., 2000. Fingering in unsaturated zone flow: a qualitative review with laboratory experiments on heterogeneous systems. Ground Water 38(6), pp. 864-871.

Tullis, B.P. and Wright, S.J., 2007. Wetting front instabilities: a three-dimensional experimental investigation. Transport in Porous Media 70, pp. 335-353.

Yiotis, A.G., Stubos, K., Boudouvis, A.G. and Yortsos Y.C., 2001. A2-D pore-network model for the drying of single-component liquids in porous media. Advances in Water Resources 24, pp. 439-460.

Yiotis, A.G., Boudouvis, A.G., Stubos, K., Tsimpanoyiannis, I.N. and Yortsos Y.C., 2004. The effect of liquid films on the drying of porous media. AIChE Journal 50, pp. 2721-2737. 